12

\title{
Умножитель субтерагерцевого диапазона на основе лампы бегущей волны с ленточным электронным пучком
}

\author{
() К.В. Белов ${ }^{1}$, А.Э. Плоских ${ }^{1,2}$, Н.М. Рыскин ${ }^{1,2, q}$ \\ ${ }^{1}$ Саратовский национальный исследовательский государственный университет, Саратов, Россия \\ ${ }^{2}$ Саратовский фрилиал Института радиотехники и электроники им. В.А. Котельникова РАН, Саратов, Россия \\ ๑ E-mail: RyskinNM@info.sgu.ru
}

Поступило в Редакцию 8 августа 2018 г.

\begin{abstract}
Представлены результаты исследования умножителя на основе лампы бегущей волны с ленточным электронным пучком и замедляющими системами в виде сдвоенной гребенки. Определены конфигурации прибора, при которых возможно получение выходной мощности свыше $60 \mathrm{~W}$ на частоте второй гармоники $190 \mathrm{GHz}$ при входной мощности около $10 \mathrm{~W}$ на частоте первой гармоники.
\end{abstract}

DOI: 10.21883/PJTF.2019.01.47149.17496

Одной из приоритетных задач современной электроники является освоение субтерагерцевого диапазона $(100-300 \mathrm{GHz})$. Компактные источники с мощностью порядка 10-100 W могут найти широкое применение в системах безопасности и противодействия терроризму, информационно-телекоммуникационных системах, спектроскопии высокого разрешения, медицине и т.д. Подобные уровни мощности в субтерагерцевом диапазоне могут обеспечить миниатюрные вакуумные усилители и генераторы [1]. В частности, в диапазоне $220 \mathrm{GHz}$ была получена выходная мощность свыше $60 \mathrm{~W}$ с помощью лампы бегущей волны (ЛБВ) с замедляющей системой (3С) в виде петляющего волновода [2]. Однако коэффициент усиления лампы был менее $15 \mathrm{~dB}$, поэтому насыщение выходной мощности наступало при высокой мощности входного сигнала (порядка $10 \mathrm{~W}$ ). Повысить коэффициент усиления и мощность можно, используя пространственно-развитые электронные пучки (ЭП) с большим поперечным сечением, например ленточные. В работе [3] была достигнута мощность около $110 \mathrm{~W}$ в ЛБВ диапазона $220 \mathrm{GHz}$ с ленточным ЭП, однако и в этом случае входная мощность была достаточно велика (порядка $1 \mathrm{~W}$ ). В субтерагерцевом диапазоне источники подобного уровня мощности, перестраиваемые в широкой полосе частот, труднодоступны. В [2,3] для этой цели использовались генераторные клистроны с распределенным взаимодействием, которые являются узкополосными. Таким образом, высокая мощность была получена только на одной или нескольких фиксированных частотах.

В связи с отмеченной проблемой привлекли внимание приборы типа умножителей частоты [4-7]. Подобный прибор состоит из трех секций, первая из которых служит для модуляции ЭП входным сигналом на частоте $\omega$. Далее пучок попадает в секцию дрейфа, где происходит группирование электронов в плотные сгустки. Ввиду нелинейного характера группировки в спектре тока происходит возбуждение высших гармонических составляющих. Сгруппированный ток возбуждает выход- ную секцию на частоте одной из высших гармоник $n \omega$, $n=2,3, \ldots$ В [4] сообщалось о разработке умножителя диапазона $170-180 \mathrm{GHz}$ на второй гармонике, а в [5] - умножителя на третьей гармонике диапазона 130-140 GHz. В этих работах использовались структуры типа петляющего волновода с круглым цилиндрическим пучком малого диаметра ( $0.25 \mathrm{~mm})$, а достигнутая экспериментально выходная мощность составляла порядка сотен милливатт. В [6] обсуждался проект умножителя третьей гармоники на базе клистрона с распределенным взаимодействием. Согласно расчетам, прибор может обеспечить мощность $15 \mathrm{~W}$ на частоте $260 \mathrm{GHz}$, однако является узкополосным: ширина полосы не превышает $90 \mathrm{MHz}$.

Как отмечалось выше, для повышения мощности целесообразно использовать пространственно-развитый ленточный ЭП. В работах [8-10] исследовалась возможность создания ЛБВ диапазона $200 \mathrm{GHz}$ с ленточным ЭП и ЗС в виде двух гребенок, сдвинутых относительно друг друга на половину периода, что обеспечивает наиболее широкую полосу усиления. Для данной ЛБВ разработана электронная пушка, создающая пучок сечением $0.1 \times 0.75 \mathrm{~mm}$ с плотностью тока свыше $100 \mathrm{~A} / \mathrm{cm}^{2}$. Согласно результатам моделирования электронно-оптической системы, при фокусировке интенсивным однородным продольным магнитным полем $1.12 \mathrm{~T}$ возможна транспортировка ЭП в канале высотой $0.2 \mathrm{~mm}$ на расстояния до $40 \mathrm{~mm}$ без оседания на стенки ЗС [9,10]. Расчеты усиления в нелинейном режиме показывают возможность получения выходной мощности порядка $60-80 \mathrm{~W}$ в режиме насыщения [10], однако мощность входного сигнала также должна быть достаточно велика $(1-10 \mathrm{~W}, \mathrm{cp}$. с $[2,3])$.

В настоящей работе обсуждаются перспективы создания умножителя на второй гармонике на основе подобного прибора. В этом случае требуется источник входного сигнала в значительно более освоенном диапазоне частот около $100 \mathrm{GHz}$. С использованием методики моделирования, развитой в [8], было выполнено проекти- 
Размеры (в $\mathrm{mm})$ 3С входной и выходной секций

\begin{tabular}{l|c|c}
\hline \multicolumn{1}{c|}{ Параметр } & Входная секция & Выходная секция \\
\hline Период & 1.03 & 0.50 \\
Толщина штыря & 0.20 & 0.10 \\
Высота штыря & 0.62 & 0.30 \\
Высота канала & 0.20 & 0.20 \\
Ширина канала & 1.70 & 0.85
\end{tabular}

рование входной секции 3С, в которой при напряжении пучка около $20 \mathrm{kV}$ обеспечивается синхронизм в достаточно широкой полосе частот. Параметры ЗС выходной секции выбирались в соответствии с данными работ [8-10]. Соответствующие характерные размеры даны в таблице. Результаты расчета электродинамических характеристик (дисперсия, сопротивление связи) обеих секций приведены в [7]. Отметим, что сопротивление связи во входной секции на частоте $95 \mathrm{GHz}$ составляет $5.2 \Omega$, а в выходной секции на частоте второй гармоники $190 \mathrm{GHz}-0.75 \Omega$.

Для численного моделирования используем хорошо известный аппарат многочастотной нелинейной теории электронно-волнового взаимодействия (см., например, [11])

$$
\begin{gathered}
\phi_{i}^{\prime \prime}=-\left(1+C \phi_{i}^{\prime}\right)^{3} \\
\times\left[\sum_{k=1}^{N_{F}} F_{k} \exp \left(k \phi_{i}\right)+i \sum_{k=1}^{N_{H}}\left(q_{k} / k\right) J_{k} \exp \left(k \phi_{i}\right)\right], \\
F_{k}^{\prime}+k\left(d_{k}-i b_{k}\right) F_{k}=-k^{2}\left(1+b_{k} C\right)^{2} \delta_{k} J_{k}, \\
J_{k}=\frac{1}{\pi} \int_{0}^{2 \pi} \exp (-i k \phi) d \phi_{0} .
\end{gathered}
$$

Здесь $\phi_{i}$ - фазы „крупных частиц“, $F_{k}$ - амплитуды гармоник высокочастотного поля, $C$ - параметр усиления Пирса, $d_{k}$ и $b_{k}$ - параметры затухания и рассинхронизма для соответствующей гармоники, $\delta_{k}-$ отношение сопротивления связи $k$-й гармоники к сопротивлению связи первой гармоники, $q_{k}=\left(\omega_{p k} / \omega C\right)^{2}$ - параметр пространственного заряда для соответствующей гармоники, $\omega_{p k}$ - плазменные частоты (здесь учтено, что разные гармоники имеют различный коэффициент редукции сил пространственного заряда), $N_{F}$ и $N_{H}$ - число учитываемых гармоник поля и тока соответственно. Штрихами обозначены производные по безразмерной координате $\xi=\beta_{e} C z, \beta_{e}=\omega / v_{0}-$ электронная постоянная распространения. При расчетах учитывается до шести гармоник тока; дальнейшее увеличение $N_{H}$ не оказывает влияния на результаты.

Уравнения (1)-(3) решаются с граничными условиями

$$
\phi(0)=\phi_{0}, \quad \phi^{\prime}(0)=0,
$$

причем начальные фазы электронов $\phi_{0}$ равномерно распределены на отрезке $[0 ; 2 \pi)$. На вход системы подается входной сигнал на частоте первой гармоники: $F_{1}(0)=F_{\text {in }}$. При этом во входной секции решается уравнение возбуждения для первой гармоники, а остальные гармоники не учитываются, так как не попадают в полосу пропускания 3С. В выходной секции решается уравнение возбуждения для второй гармоники. В секции дрейфа высокочастотное поле вообще отсутствует: $F_{1,2}=0$.

Уравнения (1)-(3) записаны в одномерном приближении, а также не учитывают скоростной разброс электронов. Однако проведенное в работе [10] сопоставление с современными пакетами 3D-моделирования KARAT и CST Particle Studio показало, что они довольно хорошо согласуются друг с другом.

В ходе численного моделирования исследовался вопрос о том, как влияют длины секций на выходную мощность. Ток пучка при расчетах выбирался равным $100 \mathrm{~mA}$, напряжение - $20 \mathrm{kV}$, а полная длина системы $-40 \mathrm{~mm}$, поскольку в соответствии с результатами моделирования электронно-оптической системы $[9,10]$ на таких расстояниях обеспечивается транспортировка пучка без оседания на стенки канала. На рис. 1 представлены зависимости выходной мощности $P_{\text {out }}$ от входной мощности $P_{\text {in }}$ при различных длинах входной секции (частоты входного и выходного сигналов равны 95 и $190 \mathrm{GHz}$ соответственно). Что касается секции дрейфа, то расчеты показали, что ее длина должна быть минимальной, и при моделировании она была принята равной $1 \mathrm{~mm}$. Из рис. 1 видно, что мощность насыщения увеличивается с уменьшением длины входной секции $l_{m}$ и соответственно с увеличением длины выходной секции $l_{\text {out }}$. При длине входной секции $5 \mathrm{~mm}$ выходная мощность превышает $60 \mathrm{~W}$ при входной мощности $11 \mathrm{~W}$. С другой стороны, при увеличении $l_{m}$ растет коэффициент преобразования $P_{\text {out }} / P_{\text {in }}$, поскольку уменьшается входная мощность, при которой достигается насыщение.

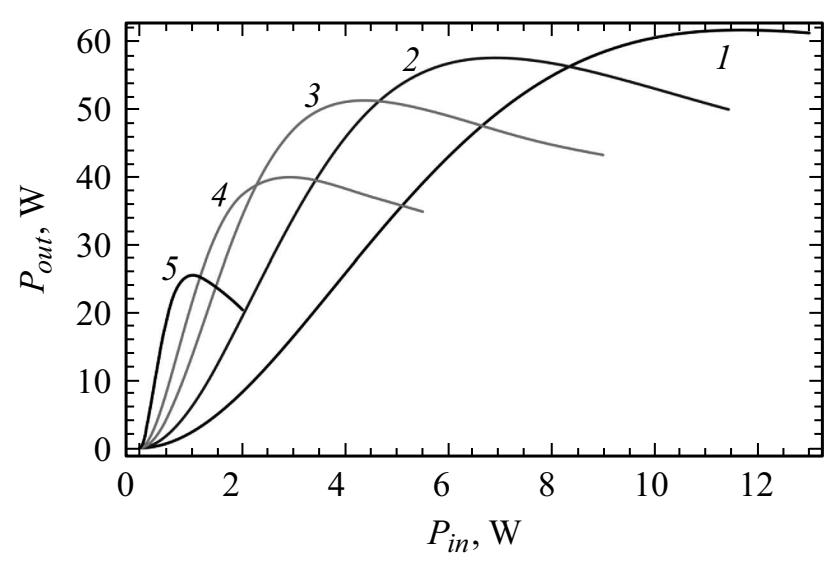

Рис. 1. Зависимости выходной мощности от входной на частоте $190 \mathrm{GHz}$ при различных длинах входной секции. $l_{i n}$, $\mathrm{mm}: 1-5,2-7,3-10,4-15,5-25$. 
На рис. 2, $a$ приведены амплитудно-частотные характеристики $\left(\mathrm{AЧX)} \mathrm{при} l_{\text {in }}=5 \mathrm{~mm}\right.$ и различных мощностях входного сигнала. При увеличении $P_{\text {in }}$ точка максимума незначительно сдвигается в область более высоких частот, что, очевидно, обусловлено эффектом нелинейного торможения пучка. Ширина полосы по уровню $-3 \mathrm{~dB}$ при $P_{i n}=11 \mathrm{~W}$ составляет более $20 \mathrm{GHz}$. Отметим, что при выбранном значении напряжения имеется еще одна точка синхронизма вблизи высокочастотной границы полосы пропускания $3 \mathrm{C} f \approx 240 \mathrm{GHz}$, поэтому на АЧХ имеется еще один небольшой максимум в окрестности этой частоты (cp. c [10]).

На рис. 2, $b$ приведены распределения мощностей первой и второй гармоник вдоль длины системы. Границы области дрейфа показаны вертикальными штриховыми линиями. Видно, что в первой секции мощность входного сигнала незначительно падает, расходуясь на модуляцию пучка. В выходной секции происходит усиление сигнала на второй гармонике, вносимого модулированным электронным пучком.
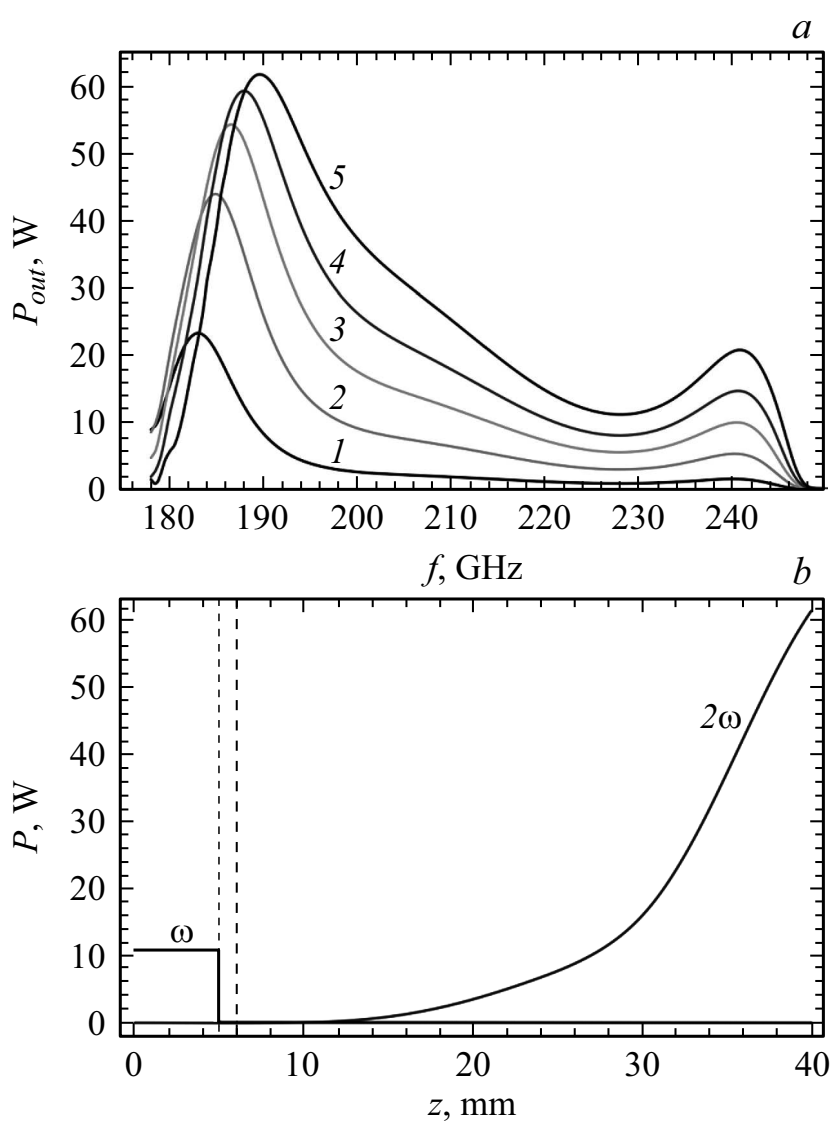

Рис. 2. Результаты моделирования при длине входной секции $5 \mathrm{~mm}$ и напряжении пучка $20 \mathrm{kV}$. $a-$ АЧХ при различной мощности входного сигнала. $P_{i n}, \mathrm{~W}: 1-2,2-4,3-6$, $4-8,5-11 . b-$ зависимости мощности первой $(95 \mathrm{GHz})$ и второй $(190 \mathrm{GHz})$ гармоник от координаты на частоте при входной мощности $11 \mathrm{~W}$.

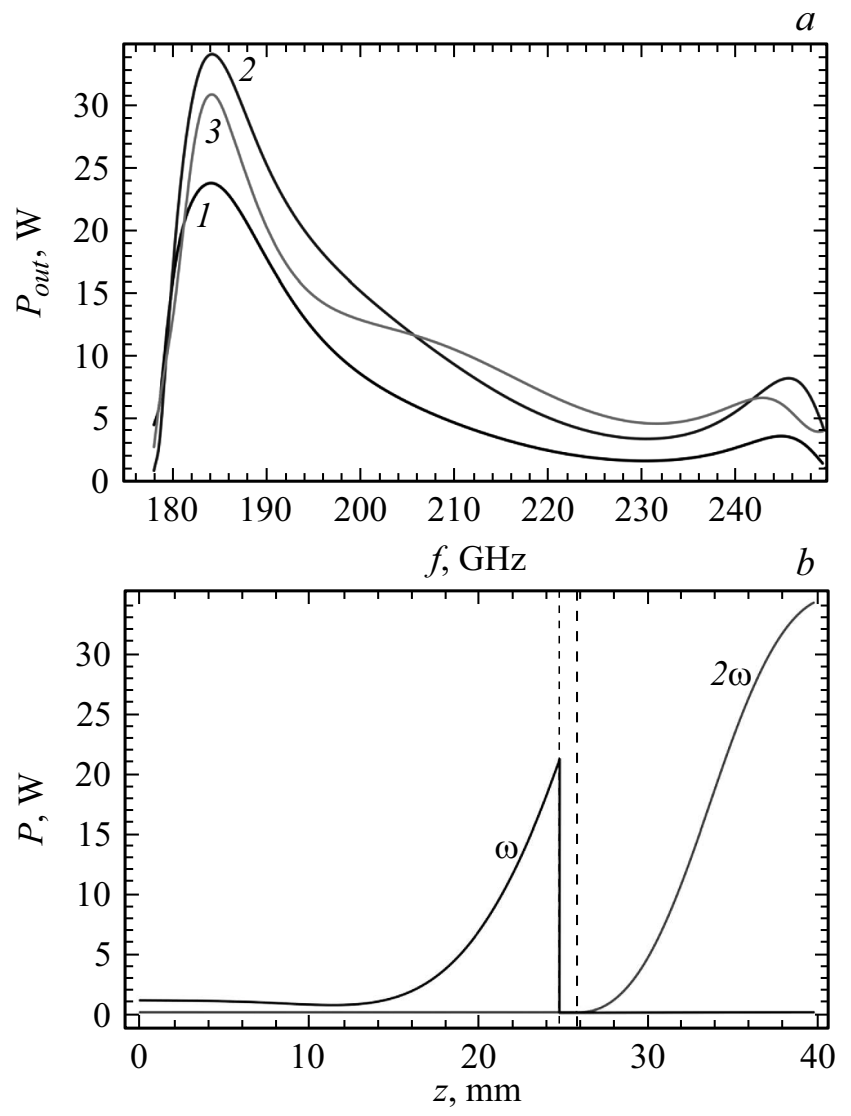

Рис. 3. Результаты моделирования при длине входной секции $25 \mathrm{~mm}$ и напряжении пучка $20 \mathrm{kV}$. $a-$ АЧХ при различной мощности входного сигнала. $P_{i n}, \mathrm{~W}: 1-0.5,2-1,3-2 . b-$ зависимости мощности первой $(92 \mathrm{GHz})$ и второй $(184 \mathrm{GHz})$ гармоник от координаты при входной мощности $1 \mathrm{~W}$.

На рис. 3 приведены аналогичные зависимости при $l_{\text {in }}=25 \mathrm{~mm}$. В этом случае максимальная выходная мощность $P_{\text {out }} \approx 34 \mathrm{~W}$ достигается при входной мощности $P_{\text {in }} \approx 1 \mathrm{~W}$, т.е. коэффициент преобразования равен 34 . Поскольку мощность меньше, чем в предыдущем случае, максимум АЧХ также достигается на несколько меньшей частоте: $f \approx 184 \mathrm{GHz}$. В первой секции происходит заметное усиление входного сигнала (рис. $3, b$ ), который также может быть выведен в полезную нагрузку.

Таким образом, в работе рассмотрена возможность создания умножителя частоты субтерагерцевого диапазона на второй гармонике с ленточным электронным пучком и ЗС в виде сдвоенных гребенок. Результаты компьютерного моделирования свидетельствуют о принципиальной возможности получения выходной мощности свыше $60 \mathrm{~W}$ и полосы по уровню $-3 \mathrm{~dB}$ около $20 \mathrm{GHz}$, для этого требуется входная мощность порядка $10 \mathrm{~W}$. Источники на основе ЛБВ такого уровня мощности в $W$ диапазоне $(92-95 \mathrm{GHz})$ представляются вполне доступными [12,13].

Работа выполнена при поддержке гранта РФФИ № 1608-00450a. 


\section{Список литературы}

[1] Kim J.I., Jeon S.G., Park G.S. Terahertz vacuum electronics // Handbook of terahertz technologies: devices and applications / Eds H.-J. Song, T. Nagatsuma. Boca Raton, FL: CRC Press, 2015. Ch. 8. P. $187-220$.

[2] Joye C.D., Cook A.M., Calame J.P., Abe D.K., Vlasov A.N., Chernyavskiy I.A., Nguyen K.T., Wright E.L., Pershing D.E., Kimura T., Hyttinen M., Levush B. // IEEE Trans. Electron Dev. 2014. V. 61. N 6. P. 1672-1678.

[3] Baig A., Gamzina D., Kimura T., Atkinson J., Domier C., Popovic B., Himes L., Barchfeld R., Field M., Luhmann N.C. // IEEE Trans. Electron Dev. 2017. V. 64. N 5. P. 2390-2397.

[4] Cai J., Wu X., Feng J. // IEEE Trans. Electron Dev. 2015. V. 62. N 2. P. $648-651$.

[5] Gong H., Wang Q., Deng D., Meng X., Dong Y., Xu J., Tang T., Su X., Wang Zh., Gong Y., Travish G. // IEEE Trans. Electron Dev. 2018. V. 65. N 6. P. 2189-2194.

[6] Makhalov P., Fedotov A. // IEEE Trans. THz Sci. Technol. 2015. V. 5. N 6. P. $1048-1052$.

[7] Karetnikova T.A., Ryskin N.M., Belov K.V., Ploskih A.E. // 2017 10th UK-Europe-China Workshop on millimetre waves and terahertz technologies (UCMMT). Liverpool, UK, 2017. P. 8068466.

[8] Рожнёв А.Г., Рыскин Н.М., Каретникова Т.А., Торгашов Г.В., Синицын Н.И., Шалаев П.Д., Бурцев А.А. // Изв. вузов. Радиофизика. 2013. Т. 56. № 8-9. С. 601-613.

[9] Каретникова Т.А., Рожнёв А.Г., Рыскин Н.М., Торгашов Г.В., Синицын Н.И., Григорьев Ю.А., Буриев А.А., Шалаев П.Д. // Радиотехника и электроника. 2016. Т. 61. № 1. C. 54-60.

[10] Karetnikova T.A., Rozhnev A.G., Ryskin N.M., Fedotov A.E., Mishakin S.V., Ginzburg N.S. // IEEE Trans. Electron Dev. 2018. V. 65. N 6. P. 2129-2134.

[11] Каи, А.М., Ильина Е.М., Манькин И.А. Нелинейные явления в СВЧ приборах О-типа с длительным взаимодействием. М.: Сов. радио, 1975. $296 \mathrm{c}$.

[12] Kowalski E.J., Shapiro M.A., Temkin R.J. // IEEE Trans. Electron Dev. 2015. V. 62. N 5. P. 1609-1616.

[13] Zhang X., Feng J., Cai J., Wu X., Du Y., Chen J., Li S., Meng W. // IEEE Trans. Electron Dev. 2017. V. 64. N 12. P. $5151-5156$. 